\title{
Retentissement de l'infection genitale a chlamydia trachomatis sur le sperme chez les hommes consultant pour infertilite du couple
}

\author{
L.AmmaR-Keskes *, R.Gdoura **, F.BouZID ***, F.Ben SalaH**, D.Sellami. **, \\ H.HAKIM. ** A.HAMmami **, T.REBAÏ *, S.REKIK ***, J.ORFILA.**** \\ * Laboratoire d'Histologie-cytologie et de Biologie de la Reproduction, Faculté de Médecine de Sfax, \\ ** Laboratoire de Bactériologie-Immunologie, CHU Habib Bourguiba, Sfax, \\ *** Service de gynécologie-obstétrique, CHU Hédi Chaker, Sfax. \\ **** Laboratoire de bactériologie-immunologie, CHU Amiens, France.
}

\section{RESUME}

Introduction: l'infection génitale à Chlamydia trachomatis (CT) est une cause fréquente d'infertilité du couple. Son retentissement sur les paramètres du sperme et sur la fertilité masculine reste controversé. Le but de cette étude prospective est d'évaluer l'impact de l'infection génitale à CT sur le sperme chez des hommes consultant pour infertilité du couple.

Matériel et méthodes : l'étude a porté sur 97 couples infertiles. La recherche de CT était réalisée sur les prélèvements génitaux par immuno-fluorescence directe (IFD), culture cellulaire, PCR et ELISA, et sur les prélèvements sanguins par micro-immunofluorescence (MTF). Chez tous les hommes, un spermogramme complet était réalisé selon la méthode standardisée de 1'OMS (1).

Résultats: $34 \%$ des hommes étaient positifs pour un ou plusieurs marqueurs de l'infection chlamydienne(CT+). Dans environ $76 \%$ des cas l'infection était asymptomatique et dans $60,6 \%$ des cas les épouses étaient CT+. Les valeurs moyennes des paramètres du spermo- gramme dans les deux groupes de patients CT-t et CT- ne montraient pas de différences significatives; elles étaient respectivement de $39,6 \% \pm 17,5 \%$ et de $40,4 \% \pm 14,9 \%$ pour la mobilité des spermatozoïdes, de $61,9 \% \pm 18,1 \%$ et $62,4 \% \pm$ $18,5 \%$ pour la vitalité, de $80,7 \times 10^{6} \pm$ $67,5 \times 10^{6}$ et $67,1 \times 10^{6} \pm 65,2 \times 10^{6}$ pour la numération et de $34,7 \% \pm 16,7 \%$ et $33 \% \pm$ $0,1 \%$ pour le taux des formes normales. L'oligospermie était significativement plus fréquente dans le groupe CT+ $(54,5 \%)$ contre $(26,9 \%)$ dans le groupe CTLes taux élevés de flagelles enroulés étaient observés chez $18,5 \%$ des patients CT+ et chez 7,4\% des patients CT-, mais la différence n'est pas significative.

Discussion: La prévalence de l'infection génitale à CT est fréquente dans notre étude. L'absence de retentissement de l'infection chlamydienne sur les valeurs moyennes des paramètres du spermogramme permet de suggérer qu'il n'existe pas d'effet délétère de CT sur les spermatozoïdes, mais nous ne pouvons pas exclure un retentissement sur leurs

Pour toute correspondance, écrire au Dr Keskes Leila, Laboratoire d'Histo-Embryologie, Faculté de Médecine, Route El Aïn, Km 0.5, Sfax, Tunisie. 
capacités fécondantes ou sur leur ultrastructure. La fréquence relativement élevée de l'oligospermie dans le groupe CT+ suggère l'existence d'un effet néfaste sur la fonction gamétogéne du testicule ou sur la perméabilité des voies spermatiques. Une autre hypothèse serait que l'oligospermie, reflet d'une spermatogenèse perturbée pourrait être associée à une diminution de l'immunité locale favorisant ainsi les infections. Des études plus rigoureuses avec une exploration complète de toutes les fonctions spermatiques et des différents compartiments du tractus génital sont nécessaires pour déterminer avec plus de précision le retentissement réel de l'infection chlamydienne sur la fonction de reproduction de l'homme.

Mots-clés : Chlamydia trachomatis, infection génitale, infertilité, spermogramme.

\section{INTRODUCTION}

Chlamydia Trachomatis (CT) est une bactérie à multiplication intracellulaire obligatoire et sexuellement transmissible. Les infections génitales à $\mathrm{CT}$ sont souvent asymptomatiques et sont très répandues dans les pays développés, touchant électivement des adultes de 15 à 25 ans. Chez l'homme, CT est responsable d'urétrites, d'épididymites et de prostato-vésiculites [23]. Alors que la salpingite chlamydienne est une cause fréquente d'infertilité chez la femme, l'impact de l'infection à Chlamydia sur les paramètres du sperme et sur la fertilité masculine reste controversé $[5,8,9$, 13, 17, 27, 29]. La détection de CT dans le sperme est classiquement effectuée par les techniques de culture cellulaire et d'immunofluorescence directe. Toutefois, la culture est souvent négative du fait de la cytotoxicité. La réaction de polymérisation en chaîne ( $\mathrm{PCR}$ ), permet actuellement une détection plus facile de l'ADN de CT et constitue avec la détermination des IgG sériques et des IgA séminales anti-CT des marqueurs indispensables dans l'exploration de la pathologie génitale masculine et dans le bilan d'infertilité $[4,15,25,28]$.

Les études réalisées à la recherche d'une éven- tuelle corrélation entre la présence de CT dans le sperme et les caractéristiques du sperme ou sa fécondance, ont montré que les effets de CT sur les paramètres du sperme n'entraînaient qu'une réduction du pourcentage de spermatozoïdes mobiles et une altération de la qualité de leur mobilité [5]. Par ailleurs, il a été montré in vitro que CT adhérait à la membrane plasmique des spermatozoïdes jouant ainsi un rôle de vecteur dans la transmission de l'infection [11, 30] et qu'il conservait son pouvoir pathogène après congélation du sperme [24].

L'objectif de notre étude était d'évaluer l'impact de l'infection génitale à CT sur les paramètres du spermogramme chez les hommes consultant pour infertilité du couple.

\section{MATERIEL ET METHODES}

\section{Patients et marqueurs de l'infection chla- mydienne:}

97 patients suivis avec leurs épouses pour infertilité du couple au service de gynéco-obstétrique du CHU Hédi Chaker de Sfax entre Janvier et Septembre 1996, ont été inclus dans l'étude. Tous les sujets étaient soumis à un questionnaire type (âge, profession, type et durée de stérilité, activité sexuelle, antécédents médicaux et chirurgicaux et urogénitaux). L'âge moyen était d'environ 36 ans (35,5 $\pm 5,1$ ) pour l'homme avec des extrêmes allant de 20 à 49 ans et d'environ 32 ans $(31,9 \pm 6,2)$ pour la femme avec des extrêmes allant de 20 à 45 ans. La durée moyenne de la stérilité était de $6,4 \pm 4$ ans. La stérilité était de type primaire dans $71,5 \%$ des cas (68/95) et de type secondaire dans les autres cas (27/95). Tous les patients ont bénéficié d'un bilan complet à la recherche d'un ou plusieurs marqueurs de l'infection à CT. Le bilan comportait :

- la recherche des anticorps sériques antiChlamydia de type IgG, IgA et IgM, par la technique de micro-immunofluorescence (MIF). La réaction était considérée positive lorsque les titres des IgG anti-CT étaient $\geq$ à 64 , ceux des $\operatorname{Ig} M$ et des $\operatorname{IgA} \geq$ à 12 sans réactions croisées avec les antigènes des deux autres espèces.

- la recherche des antigènes de CT dans le 
sperme par la technique d'immunofluorescence directe (IFD) utilisant un anticorps monoclonal anti-CT marqué à l'isothiocynate de fluoresceine (test direct Micro Trak Syva). L'IFD était considérée positive lorsqu'au moins 10 particules typiques de CT étaient observées.

- la recherche des IgA anti-CT dirigés contre l'antigène liposacchardique, dans le liquide séminal et dans le prélèvement urétral par la technique ELISA (kit commercial : r ELISA medac, Diagnostika Germany).

- la mise en évidence de CT dans le prélèvement urétral par culture sur cellules de Mc Coy. La culture était considérée positive lorsqu'une ou plusieurs inclusions intracellulaires caractéristiques de CT était(ent) observée(s).

- la mise en évidence de l'ADN chlamydien par PCR (Cobas amplicor, Roche) dans le sperme et dans le prélèvement urétral.

Chez les femmes, outre le prélèvement sanguin, un prélèvement endocervical était systématiquement effectué à la recherche des IgA sécrétoires anti-CT par ELISA ainsi qu'à la recherche de CT par IFD, PCR et par culture cellulaire.

Pour des raisons pratiques, tous les patients ayant au moins un marqueur de l'infection chlamydienne déterminé par les techniques précédentes, étaient considérés atteints de chlamydiose génitale, quel que soit le type de prélèvement (génital ou sanguin).

\section{Analyse du sperme}

Le sperme était prélevé au laboratoire par masturbation et dans un flacon stérile, après un délai d'abstinence d'environ 3 jours. Il servait pour la pratique d'un spermogramme et d'une spermoculture à la recherche de germes banals.

Le spermogramme était réalisé 30 minutes environ après le prélèvement, sur sperme liquéfié. Nous avons utilisé la technique standardisée ainsi que les critères de normalité (Tableau I) recommandés par l'OMS [1]. Le spermogramme comportait un examen macroscopique du sperme qui analysait l'aspect, le volume et la viscosité, et des examens micro-
Tableau I : Paramètres du spermogramme selon les normes officielles de l'OMS (1)

\begin{tabular}{lll} 
Paramètres & $\begin{array}{l}\text { Normes } \\
\text { (OMS) }\end{array}$ & $\begin{array}{l}\text { Définition de } \\
\text { l'anomalie }\end{array}$ \\
\hline Volume $(\boldsymbol{m l})$ & $\geq 2$ & $\begin{array}{l}<\mathrm{ml}: \text { hypo- } \\
\text { spermie } \\
\end{array}$ \\
& & $>2 \mathrm{ml}:$ hyper \\
& spermie
\end{tabular}

\begin{tabular}{|c|c|c|}
\hline \multirow[t]{2}{*}{$\begin{array}{l}\text { Numération } \\
\left(10^{6 / m l}\right)\end{array}$} & $20-200$ & $\begin{array}{c}0 \text { : azoospermie } \\
<20 \text { : oligozoo- } \\
\text { spermie }\end{array}$ \\
\hline & & $\begin{array}{l}>200: \text { : poly- } \\
\text { zoospermie }\end{array}$ \\
\hline
\end{tabular}

Mobilité totale $\geq 50$

$(\%)$

$<50 \%$ : asthé nozoospermie

\begin{tabular}{|c|c|c|}
\hline $\begin{array}{l}\text { Morphologie } \\
\text { normale (\%) }\end{array}$ & $\geq 30$ & $\begin{array}{l}<30 \% \text { : térato- } \\
\text { zoospermie }\end{array}$ \\
\hline Vitalité (\%) & $\geq 75$ & $\begin{array}{l}<75 \% \text { : nécrozoo- } \\
\text { spermie }\end{array}$ \\
\hline $\begin{array}{l}\text { Leucocytes } \\
\left(10^{6} / \mathrm{ml}\right)\end{array}$ & $<1$ & $\geq 1$ : pyospermie \\
\hline
\end{tabular}

scopiques, incluant une analyse détaillée des paramètres cytomorphologiques des spermatozoïdes (agglutination spontanée, mobilité, numération, vitalité et morphologie). La mobilité était déterminée par l'examen au microscope optique à contraste de phase d'une goutte calibrée de sperme, déposée entre lame et lamelle et observée à l'objectif x 40 (grossissement 400). Pour chaque sperme, deux valeurs de la mobilité étaient notées : la première, la mobilité totale (Mob 30), détermine le pourcentage des spermatozoïdes mobiles et, la deuxième détermine le pourcentage des spermatozoïdes ayant un mouvement progressif et rapide (PR 30). L'asthénospermie était considérée modérée lorsque Mob 30 était comprise entre $30 \%$ et $50 \%$; elle était considérée sévère lorsque Mob 30 était $\leq 20 \%$ et / ou PR $30 \leq$ $10 \%$. La vitalité était évaluée par le test de Williams [1]. La nécrospermie était considérée modérée lorsque le pourcentage de spermatozoïdes vivants était compris entre $50 \%$ et $75 \%$ 
$(50 \% \leq$ vit $\leq 75 \%)$; elle était considérée comme sévère pour les valeurs plus faibles (Vit $\leq$ $50 \%$ ). La morphologie était étudiée sur des frottis de sperme colorés par le Schorr. Le spermogramme comportait également la détection des leucocytes dans le liquide séminal par la méthode de révélation histochimique de la peroxydase à la phloxine-benzidine.

\section{Méthode statistique}

Les valeurs des paramètres du spermogramme sont exprimées en moyenne plus ou moins l'écart type. Le test de signification utilisé pour la comparaison des moyennes et des pourcentages est celui de l'écart réduit (loi de Laplace Gauss). La signification est donnée au risque de $5 \%$.

\section{RESULTATS}

\section{Marqueurs de l'infection chlamydienne}

Les résultats obtenus par les différentes techniques de mise en évidence de l'infection chlamydienne montrent que $34 \%$ (33/97) des hommes étaient positifs pour un ou plusieurs marqueurs (CT+) (Tableau II). Les trois quarts (25/33) de ces patients CT+ étaient totalement asymptomatiques et ne présentaient aucun signe génito-urinaire. Dans $60,6 \%$ des cas (20/33), les deux partenaires présentaient des signes biologiques de l'infection chlamydienne (Tableau II). La recherche des IgA et IgM sériques par MIF était toujours négative. Les spermocultures recherchant les germes banals étaient également toutes négatives. $38 \%$ des épouses (37/97) étaient CT+ ; 46\% (17/37) d'entre elles avaient des partenaires CT-.

\section{Le spermogramme}

Parmi les 97 patients inclus dans cette étude, 8 avaient une azoospermie d'origine non déterminée. 3 d'entre eux présentaient des signes biologiques de l'infection chlamydienne.

Les valeurs moyennes des paramètres du spermogramme des deux groupes de patients CT+ et CT- figurent dans le tableau III. Il n'existe pas de différence significative entre les deux groupes. Le volume moyen du sperme ainsi que le taux moyen des spermatozoïdes de formes typiques et la numération étaient normaux dans les 2 groupes. Seules la mobilité et la vitalité étaient anormales dans les deux groupes (selon les normes de l'OMS). L'asthénospermie et la nécrospermie étaient modérées.

Lagglutination spontanée des spermatozoïdes était présente chez $43,3 \%$ (13/30) des patients CT+ et chez $40,6 \%$ (24/59) des patients CT-

L'analyse comparative plus fine des principaux paramètres du spermogramme entre les deux groupes de patients CT+ et CT-, ne montre pas de différences significatives entre les deux groupes de patients CT+ et CT- pour les fréquences de l'hyperspermie, de la mobilité normale et de la vitalité normale, pour les degrés de l'asthénospermie, de la nécrospermie ainsi que pour la concentration des leucocytes (Tableaux IV et V). Seule l'oligospermie était significativement plus fréquente dans le groupe CT+ (Tableau V). Chez les époux CT+ et CT- des femmes CT+, les numérations étaient respectivement de $77,8 \times 10^{6} \pm 64,8 \pm$ $10^{6}$ et de $50,3 \times 10^{6} \pm 63,6 \times 106$ (différence non significative).

\section{DISCUSSION}

La chlamydiose génitale est une cause fréquente d'infertilité du couple. Sa prévalence est élevée chez l'homme puisqu'elle est responsable de $10 \%$ à $40 \%$ des uréthrites non gonococciques (21). La détection de l'infection génitale à CT se heurte à des difficultés techniques. Néanmoins, son dépistage est justifié chez les jeunes sexuellement actifs en raison de la transmission sexuelle du germe et de ses effets sur l'appareil génital, mais aussi en raison de l'efficacité du traitement antibiotique et du caractère asymptomatique de l'infection. Dans cette étude, nous avons utilisé les techniques d'immunofluorescence directe, de culture cellulaire, de PCR ainsi que les techniques de micro-immunofluorescence et d'ELISA afin de détecter la chlamydiose génitale chez les hommes consultant pour infertilité du couple et de déterminer son retentissement sur les paramètres du spermogramme. 
Tableau II : Résultats positifs des différentest techniques de mise en évidence de l'infection chlamydienne chez les deux partenaires du groupe CT+.

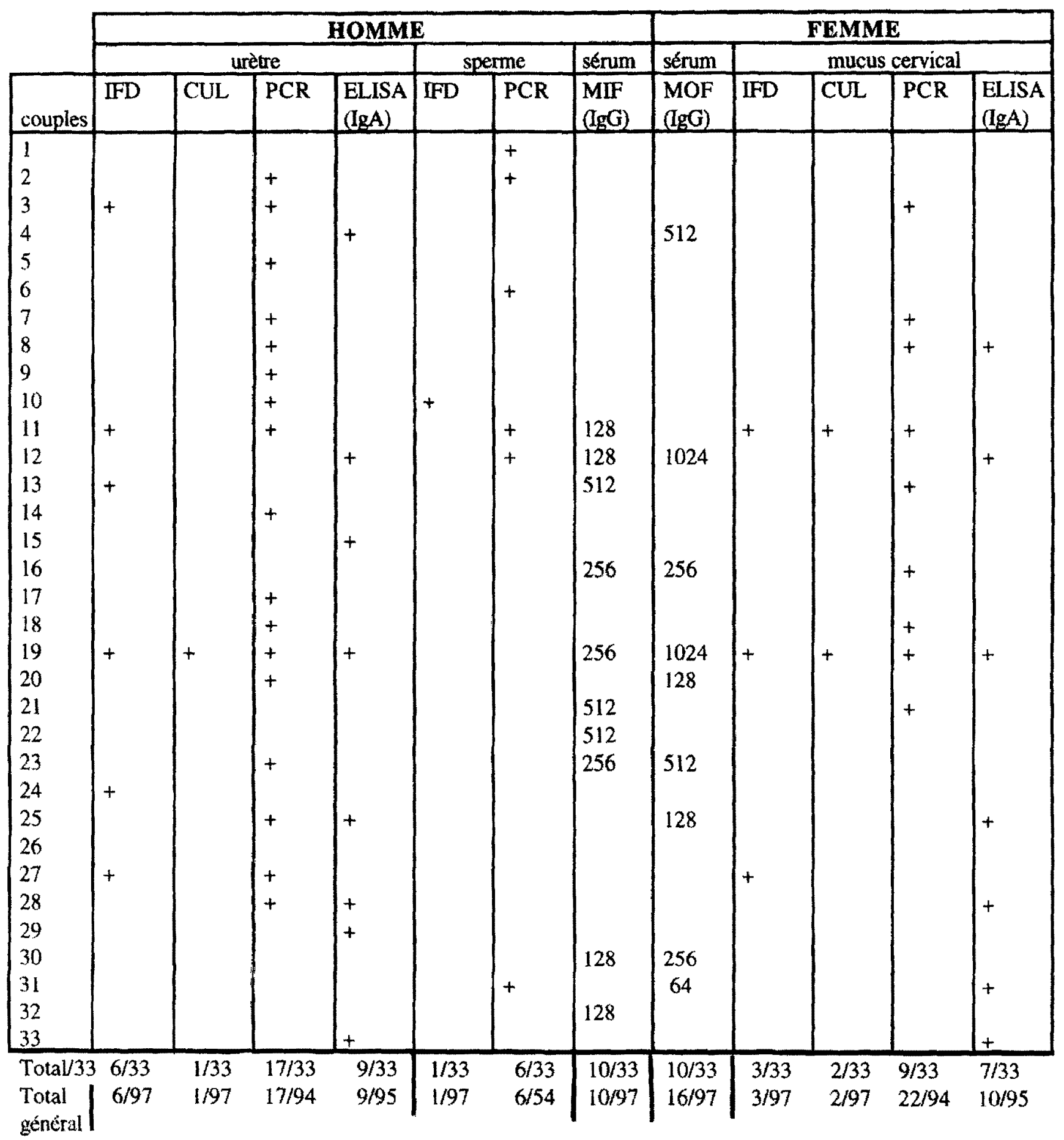


Tableau III Valeurs moyennes et extrêmes des paramètres du sperme chez les patients des deux groupes, Chlamydia + et Chlamydia -

\begin{tabular}{|c|c|c|c|c|c|c|c|}
\hline & \multicolumn{2}{|l|}{ Chlamydia + } & \multicolumn{2}{|c|}{ Chlamydia - } & \multirow[b]{2}{*}{ Min } & \multirow[b]{2}{*}{$\operatorname{Max}$} & \multirow{2}{*}{$\begin{array}{c}\text { Test } \\
\text { statistique }\end{array}$} \\
\hline & Moy \pm DS & Min & Max & Moy $\pm D S S$ & & & \\
\hline Volume (ml) & $3,2 \pm 1,3$ & 0,6 & 8,5 & $3,5 \pm 2,1$ & 1 & 13,2 & NS \\
\hline $\begin{array}{l}\text { Mobilité Total } \\
(\text { Mob30) }(\%)\end{array}$ & $39,6 \pm 17,5$ & 5 & 70 & $40,4 \pm 14,9$ & 10 & 65 & NS \\
\hline $\begin{array}{l}\text { Mobilité Progressive } \\
\text { et rapide (PR30)(\%) }\end{array}$ & $12,1 \pm 7,9$ & 0 & 25 & $12,5 \pm 9,4$ & 0 & 55 & NS \\
\hline Numération (106/) & $80,7 \pm 67,8$ & 0,36 & 243,4 & $67,1 \pm 65,2$ & 0,9 & 290 & NS \\
\hline Vitalité (\%) & $61,9 \pm 18,1$ & 25 & 82 & $62,4 \pm 18,5$ & 18 & 90 & NS \\
\hline $\begin{array}{l}\text { Formales } \\
\text { normales }(\%)\end{array}$ & $34,7 \pm 16,7$ & 3 & 64 & $33 \pm 15$ & 5 & 73 & NS \\
\hline IAM & $1,4 \pm 0,1$ & 1,1 & 1,7 & $1,3 \pm 0,1$ & 1,1 & 1,7 & NS \\
\hline Leucocytes $(103 / \mathrm{ml})$ & $274 \pm 643,9$ & 16 & 3280 & $429,1 \pm 1.186,9$ & 80 & 6040 & NS \\
\hline
\end{tabular}

Tableau IV: Distribution détaillée de certains paramètres du spermogramme dans les deux groupes CT + et CT .

\begin{tabular}{cccc}
\hline & Chlamydia $+(\%)$ & Chlamydia $-(\%)$ & Test statistique \\
volume (ml)>6 & $1 / 31(3,2)$ & $9 / 63(14,2)$ & $\mathrm{NS}$ \\
Mob 30 (\%) $\geq 50$ & $10 / 33(30,3)^{*}$ & $16 / 63(25,3)^{*}$ & $\mathrm{NS}$ \\
$\leq 20$ & $9 / 33(27,2)^{*}$ & $11 / 62(17,7)^{*}$ & $\mathrm{NS}$ \\
PR 30 (\%) $\geq 25$ & $5 / 33(15,1)^{*}$ & $6 / 62(9,6)^{*}$ & $\mathrm{NS}$ \\
$\leq 10$ & $20 / 33(60,6)^{*}$ & $38 / 62(61,2)^{*}$ & $\mathrm{NS}$ \\
vitalité $(\%) \geq 75$ & $7 / 25(28)$ & $18 / 54(33,3)$ & $\mathrm{NS}$ \\
$50 \leq \mathrm{vit}<75$ & $12 / 25(48)$ & $21 / 54(38,8)$ & $\mathrm{NS}$ \\
$<50$ & $6 / 25(24)$ & $15 / 54(27,7)$ & $\mathrm{NS}$ \\
\hline
\end{tabular}


Tableau V : Distribution des concentrations de spermatozoüdes et de PNN dans les spermes des deux groupes $\mathrm{CT}+$ et $\mathrm{CT}$ -

Chlamydia $+(\%) \quad$ Chlamydia $-(\%) \quad$ Test statistique

\begin{tabular}{cccc}
\hline Numération $(\mathbf{1 0 6 / \mathrm { ml } )}$ & & & \\
$<20$ & $18 / 3354,5)^{*}$ & $17 / 63(26,9)^{*}$ & $\mathrm{~S}(\mathrm{p}<0,05)$ \\
$\leq 10$ & $7 / 33(21,2)^{*}$ & $13 / 63(20,6)^{*}$ & $\mathrm{NS}$ \\
$\leq 1$ & $5 / 33(15,1)^{*}$ & $6 / 63(9,5)^{*}$ & $\mathrm{NS}$
\end{tabular}

leucocytes $(103 / \mathrm{ml})$

$\begin{array}{llcc}\geq 500 & 5 / 33(15,1) & 8 / 55(14,5) & \text { NS } \\ <500 & 28 / 33(84,8) & 47 / 55(85,4) & \text { NS } \\ \geq 1000 & 1 / 33(3) & 6 / 55(10,9) & \text { NS }\end{array}$

* Azoospermie incluse

$34 \%$ des hommes inclus dans cette étude, avaient au moins un marqueur positif de l'infection chlamydienne (CT+). Dans environ trois quart des cas, l'infection était asymptomatique. Cette prévalence est comparable à celle rapportée par Steven et coll [25] qui ont trouvé la chlamydiose chez $39,3 \%$ des patients infertiles, par PCR. Par contre, dans l'étude de Dieterle et coll [6], la prévalence de l'infection à CT asymptomatique, détectée chez des patients infertiles par PCR ou par hybridation in situ, était seulement de $20 \%$. Cette valeur est proche de celle rapportée par Radouani et coll [22] qui trouvèrent des marqueurs sérologiques spécifiques à CT chez $24,5 \%$ des hommes stériles. Aynaud et coll [2] ont rapporté des valeurs beaucoup plus basses : $1,2 \%$ par la culture cellulaire et 5\% par PCR Amplicor, chez des patients consultant en andrologie pour des motifs divers (infection génitale, symptomatologie uro-génitale etc ...). La prévalence relativement élevée de la chlamydiose chez les hommes infertiles est un argument en faveur de l'implication de cette infection dans la pathogénie de l'infertilité. Cette prévalence est également élevée chez les femmes, puisque $38 \%$ des épouses incluses dans cette étude étaient également CT+. Dans environ un cas sur deux, les deux partenaires étaient CT+ (60\% des épouses des hommes CT+ et $54 \%$ des époux des femmes CT+). Ces résultats sont en faveur du caractère sexuellement transmissible de la maladie. Le fait que dans environ la moitié des cas, l'infection ne touchait qu'un seul partenaire pourrait s'expliquer par l'hypothèse selon laquelle la transmission sexuelle de la bactérie dépendrait de l'ancienneté, donc de l'évolutivité de la maladie et des défenses immunitaires locales du partenaire sain.

L'analyse des paramètres du spermogramme chez les 89 patients infertiles non azoospermiques montre l'existence d'une asthénospermie et une nécrospermie modérées dans les deux groupes de patients CT+ et CT-. Nous avions trouvé précédemment (non publié) que ces deux anomalies existaient chez la majorité des patients infertiles et nous avons suggéré qu'elles pourraient être dues à des facteurs toxiques liés à l'environnement et aux conditions de travail, plutôt qu'à des infections du tractus génital. L'atteinte de la mobilité n'était pas seulement quantitative mais aussi qualitative puisque les taux moyens de spermatozoïdes progressifs et rapides étaient faibles dans les deux groupes, CT+ et CT(Tableau III). Diquelou et coll [5] ont décrit un mouvement de "jerk" dans les spermatozoïdes d'homme atteints de chlamydiose ; ce phénomène était observé dans $71 \%$ des cas et correspondait à un mouvement lent avec un trajet sinueux survenant en alternance avec une trajectoire et une vitesse normales. L'altération de la mobilité des spermatozoïdes est rapportée par d'autres auteurs [6, 28]. Cependant, dans certaines études comparant les caractéristiques du sperme en fonction de la 
sérologie antichlamydienne, il n'a pas été retrouvé de différence significative entre des hommes séropositifs et séronégatifs pour les $\mathrm{AC}$ anti-CT $[6,10,12]$.

Comme Bjercke et coll [4] qui n'ont rapporté aucun effet sur le volume de l'éjaculat ou sur la concentration de spermatozoïdes d'hommes inféconds en présence d'IgA anti CT dans le plasma séminal, par comparaison avec des hommes inféconds sans anticorps IgA, les valeurs moyennes du volume d'éjaculat, de la numération des spermatozoïdes, des formes normales et de la leucospermie dans notre étude, ne montraient aucune différence significative entre les deux groupes de patients CT+ et CT-. Diquelou et coll [7] ont constaté le même résultat pour deux groupes de patients IgA positifs et IgA négatifs dans le prélèvement urétral. Dans l'étude de Close et coll [5], il n'existait pas de différence du nombre de leucocytes entre les hommes avec ou sans $\mathrm{AC}$ anti-CT sériques. Waltraud et coll [27], n'ont trouvé aucune différence entre les deux groupes CT+ et CT- pour les deux paramètres suivants : morphologie et mobilité. L'ensemble de ces résultats permet de suggérer que CT n'exerce aucun effet spécifique apparent sur les spermatozoïdes et sur le sperme. Cependant, sans l'étude des marqueurs biochimiques spécifiques des fonctions glandulaires et épididymaires et sans les tests complémentaires de l'exploration fonctionnelle des spermatozoïdes, il serait hatif d'exclure un éventuel retentissement de CT sur le pouvoir fécondant ou l'ultrastructure des spermatozoïdes. En effet, il a été montré que CT pouvait adhérer aux spermatozoïdes et pénétrer dans la tête et le flagelle du spermatozoïde [26]. L'infertilité dans les infections chlamydiennes peut être aussi le résultat de l'inactivation des spermatozoïdes par les anticorps anti-spermatozoïdes [28]. Dans notre étude, nous n'avons pas recherché les anticorps anti-spermatozoïdes dans le sperme, mais l'agglutination spontanée, évocatrice de l'immunisation anti-spermatozoïdes, n'était pas significativement plus fréquente dans le groupe $\mathrm{CT}+(43,3 \%$ contre 40,67\%). Dans l'étude de Witkin et coll [28], les anticorps anti-spermatozoïdes étaient présents chez tous les patients $\mathrm{CT}+$ diagnos- tiqués par PCR, alors qu'ils étaient absents chez tous les patients PCR-; ces auteurs ont suggéré que la présence des anticorps antispermatozoïdes associée à la mauvaise qualité du sperme chez les patients CT+ asymptomatiques, pouvait être le reflet d'une réaction immunitaire localisée dans le système génital masculin [28]. La réponse autoimmune anti-spermatozoïde locale est associée à une activation et une prolifération des lymphocytes Tgd [19]. Cette activation des lymphocytes parait être une source de production de protéines de choc thermique (HSP60) dont la présence dans le sperme est corrélée avec la présence d'anticorps antispermatozoïdes et d'anticorps anti-chlamydia (IgA) [20].

L'analyse plus fine des paramètres du spermogramme (tableaux IV, V et VI) ne montre pas de différences significatives entre les patients CT+ et CT-pour les fréquences et les degrés de l'asthénospermie et de la nécrospermie. Diquelou et coll [7] ont rapporté une plus grande fréquence de l'asthénozoospermie $(\mathrm{M}<40 \%)$ chez les patients $\mathrm{CT}+$ par culture de prélèvement urétral, par rapport à CT-. Dans l'étude de Dieterle et coll [6], la mobilité était anormale dans $41 \%$ des cas, mais aucun effet n'a été rapporté sur la vitalité. Dans celle de Witkin et coll [28], 36,4\% (4/11) des hommes PCR positifs étaient asthénospermiques contre 5,9\% (1/17) des hommes PCR négatifs. La seule différence significative observée dans notre étude concernait l'oligospermie qui était nettement plus fréquente chez les patients CT+, ce qui constitue un argument en faveur de l'atteinte testiculaire avec une altération de la spermatogénèse ou encore des voies spermatiques ; celles-ci seraient partiellement obstruées par des remaniements tissulaires secondaires à la réaction inflammatoire engendrée par CT. Une autre hypothèse pourrait être que l'oligospermie, reflet d'une spermatogénèse perturbée, serait associée à une diminution des défenses immunitaires locales favorisant ainsi les infections. La comparaison des numérations chez les époux CT+ et CT- des femmes CT+ ne montre aucune différence significative. Aucune modification de la concentration des spermatozoïdes n'a été 
Tableau VI : Distribution des anomalies morphologiques du sperme et valeurs de l'IAM chez les patients CT + et CT -

\begin{tabular}{|c|c|c|c|}
\hline & Chlamydia $+(\%)$ & Chlamydia $-(\%)$ & Test statistique \\
\hline Formes nles $(\%) \leq 30$ & $9 / 29(31)$ & $25 / 58(39,6)$ & NS \\
\hline$<10$ & $3 / 29(10,3)$ & $4 / 58(6,8)$ & NS \\
\hline \multicolumn{4}{|c|}{ Fl.Enroulés (pour 100spz) } \\
\hline$<10$ & $12 / 27(44,4)$ & $30 / 54(55,5)$ & $\mathrm{NS}$ \\
\hline$\geq 20$ & $>5 / 27(18,5)$ & $4 / 54(7,4)$ & NS \\
\hline \multicolumn{4}{|c|}{ Index d'anomalies multiples IAM } \\
\hline$\geq 1,5$ & $9 / 26(34,6)$ & $13 / 57(22,8)$ & NS \\
\hline$\leq 1,3$ & $11 / 26(42,3)$ & $16 / 57(28)$ & NS \\
\hline
\end{tabular}

observée dans l'étude de Witkin et coll [28]. Du point de vue morphologique, le degré de la tératospermie n'était pas différent entre les deux groupes CT+ et CT-. Par ailleurs, l'anomalie du flagelle enroulé n'était pas plus fréquente chez les patients $\mathrm{CT}+$. Il a été longtemps admis que cette anomalie est fréquemment associée à une bactériospermie ; elle a été également constatée au cours des infections expérimentales [3]. Cependant, cette notion est actuellement controversée et il a été rapporté que la présence de nombreux flagelles enroulés n'est pas particulièrement indicatrice d'une infection du sperme (18). Jouannet et coll [14] ont rapporté que la valeur la plus prédictive de conception chez les couples infertiles était donnée par l'IAM. Quand l'IAM était > 1,6, le taux de grossesse était significativement plus bas, comparativement aux valeurs plus basses de l'IAM. Dans notre étude; les valeurs élevées de l'IAM $(\geq 1,5)$ n'étaient pas significativement plus fréquentes dans le groupe CT + .

En conclusion, nous avons montré dans notre étude que la prévalence de l'infection chlamydienne chez les hommes infertiles était relativement élevée et que la chlamydiose génitale affectait souvent les deux partenaires. La présence de chlamydia trachomatis dans les voies génitales n'altère pas vraisemblablement de manière spécifique la qualité du sperme ; cependant, l'infection chlamydienne entraînerait des remaniements importants du tractus génital se compliquant d'une réduction de la production de spermatozoïdes ou d'une diminution de la perméabilité des voies spermatiques, réduisant leur expulsion au cours de l'éjaculation. Elle pourrait être également la conséquence d'une diminution des défenses locales associées aux troubles de la spermatogénèse. Toutes ces hypothèses méritent d'être confirmées par des études comparatives plus complètes, explorant les fonctions de tous les compartiments du tractus génital masculin en présence et en absence de l'infection chlamydienne. Enfin, comme cela a été souligné dans des études antérieures $[4,15,25,28]$, l'utilisation dans cette étude de plusieurs techniques de détection de l'infection chlamydienne nous a permis de montrer l'importance et l'intérêt de la PCR, ainsi que la recherche des IgG sériques par MIF et des IgA locales par la technique d'ELISA dans la détection de l'infection chlamydienne. Ces techniques constituent des marqueurs nécessaires et très informatifs dans l'exploration de la pathologie génitale masculine et dans le bilan d'infertilité. La culture cellulaire et l'IFD se heurtent à des difficultés techniques et donnent beaucoup de résultats faussement négatifs. 


\section{REFERENCES}

1. AUGER J., JOUANNET P. : Valeurs de référence des caractéristiques du sperme. In : Auger J., Jouannet P., Ed INSERM. Manuel de laboratoire de l'OMS. Analyse du sperme humain et de l'interaction des spermatozoïdes avec le mucus cervical, Paris, 1993 : $45-46$.

2. AYNAUD O., BIJAOUI G., CASANOVA JM. et al. : Prévalence de Chlamydia trachomatis parmi les hommes consultant en andrologie. Etude comparative entre la culture cellulaire et la PCR sur le sperme. J. Gynecol. Obstet. Biol. Reprod. 1996, $25:$ 479-484.

3. BISSON JP., CZYCLIK F. : Retentissement de l'infection génito-urinaire sur les spermatozoïdes. J. Urol. Nephrol., 1974, 7-8 : 631-636.

4. BJERCKE S., PURVIS K. : Chlamydial serology in the investigation of infertility. Hum. Reprod., 1992, 7 : 621-624.

5. CLOSE CE., WANG SP., ROBERTS PL. et al. : The relationship of infection with Chlamydia trachomatis in the parameters of male fertility and sperm autoimmunity. Fertil. Steril., 1987, $48: 880-3$.

6. DIETERLE S., MAHONY JB., LUINSTRA KE. et al. : Chlamydial immunoglobulin IgG and IgA antibodies in serum ans semen are not associated with the presence of chlamydia trachomatis DNA or rRNA in semen from male partners of infertile couples. Hum. Reprod., 1995, vol 10, $\mathrm{n}^{\circ} 2:$ 315-319.

7. DIQUELOU JY., PARTORINI P., FENEUX D. et al. : Le rôle de chlamydia trachomatis dans la survenue de mouvements anormaux des spermatozoïdes. J. Gynecol. Obst. Biol. Reprod., 1989, 18 : 615-625.

8. DOLIVO M., ASKIENAZY-ELBHAR M. : Rôle de l'infection chlamydienne chronique dans l'hypofertilité masculine. Infetiologie, $1993, n^{\circ} 47$, Sept.

9. EGGERT-KRUSE W., GERHARD J., NAHER H. et al. : Chlamydial infection - a female and / or male infertility factor? Fertil. Steril., 1990, $53:$ 1037-1043.

10. EGGERT-KRUSE W., BUHLINGER-GOPFARTH N., ROHR G. et al. : Relationship of antibodies to Chlamydia trachomatis in ejaculates and potential seminal infection markers in asymptomatic subfertile males. In : Coden HUREE eds. Human Reproduction, 12th Annual Meeting, Maastricht, June 30 - July 3, 1996, Oxford University Press, 1996, Vol 11, Abstract Book $1: 97$.

11. FRIBERG J., GLEICHER N., SUAREZ M. et al. : Chlamydia attached to spermatozoa. J. inf. diseases, 1985, 152, $4: 852$.

12. GREENDALE GA., HAAS ST., HOLBROOK K. et al.: The relationship of chlamydia trachomatis

13. HELLSTROM WJG., SCHACHTER J., SWEET RL. et al: Is there a role fore chlamydia trachomatis and genital mycoplasma in male infertility ? Fertil. Steril., 1987, 48, 2 : 337-339.
14. JOUANNET P., DUCOT B., FENEUX D. et al. : Male factors and the likehood of pregnancy in infertile couples. I study of sperm characteristics. In. J. Androl., 1988, $11: 379$.

15. KOJIMA H., WANG SP., KUO CC. et al. : Local antibody in semen for rapid diagnosis of chlamydia trachomatis epididymitis. J. Uro., 1988, 140 : 528-531.

16. MARDH PA., COLLEN S., SYLWAN J. : Inhibitory effects on the formation of chlamydia inclusions in Mc Coy cells by seminal fluid and some of its components. Invest. Urol., 1980, $17: 510-515$.

17. MIEUSSET R. : Germes à tropisme cellulaire (Chlamydiae, Mycoplasmes) et perturbation de la fécondance du sperme. Andrologie, 1994, $4:$ 406-413.

18. MARMOR D. : Le spermogramme. Contracept. Fertil. Sex., 1994, vol. 22, $\mathrm{n}^{\circ} 9$ : 508-511.

19. MUNOZ MG., WITKIN SS. : Autoimmunity to spermatozoa, asymptomatic chlamydia trachomatis genital tract infection and gd T lymphocytes in seminal fluid from the male partners of couples with unexplained infertility. Hum. Reprod., 1995, vol. 10, $\mathrm{n}^{\circ} 5$ : 1070-1074.

20. MUNOZ MG., JEREMIAS J., WITKIN SS. : The 60 heat shock protein (HSP60), antisperm antibodies and undetected chlamydia trachomatis infection in semen from male partners of couples with undiagnosed infertility. In : Coden HUREE eds. Human Reproduction, 12th Annual Meeting, Maastricht, June 30 July 3, 1996, Oxford University Press, 1996, Vol. 11, Abstract Book 1 : 189-190.

21. ORIEL JD. : Male genital chlamydia Trachomatis infections. J. Infect., 1992, Jul., Vol. 25, Suppl. 1 : 357.

22. RADOUANI F., BENNANI A., TAKOURT B. et al. : Chlamydiose et infertilité masculine au Maroc. Contracept. Fertil. Sex., 1996, vol. 24, nº $10: 779-$ 783.

23. SCHOUMAN M. : Infections génitales à Chlamydiae et Mycoplasmes. Andrologie, 1994, $4:$ 403-405.

24. SHERMAN JK., JORDAN GW. : Cryosurvival of chlamydia trachomatis during cryo-preservation of human spermatozoa. Fertil. Steril., 1985, 43 : 664-6.

25. STEVEN S., WITKIN SS., JAN JEREMIAS AB. et al. : Detection of chlamydia trachomatis in semen by the polymerase chain reaction in male members of infertile couples. Am. J. Obstet. Gynecol., 1993, May : 1457-1462.

26. TURKAN E. : Ultrastructural observations on the entry of chlamydia trachomatis into human spermatozoa. Hum. Reprod., 1993, vol. $n^{\circ} 3$ : 416-421.

27. WALTRAUD EK., NICOLA BG., GERHARD R. et al.: Antibodies to chlamydia trachomatis in semen and relationship with parameters of fertility. Human Reprod., 1996, vol. 11, $\mathrm{n}^{\circ} 7:$ 1408-1417.

28. WITKIN SS., JEREMIAS J., GRIFO JA. et al. : 
Detection of chlamydia trachomatis in semen by the polymerase chain reaction in male members of infertile couples. Am. J. Obstet. Gynecol., 1993, 168 : 1457-1462.

29. WOLFF H., NEUBERT U., ZEBHAUSER M. et al. : Chlamydia trachomatis induces an inflammatory response in the male genital tract and is associated with altered semen quality. Fertil. Steril., 1991, 55 : 1017 101.

30. WOLNER-HANSSEN P., MARDH PA. : In vitro tests of adherence of chlamydia to human spermatozoa. Fertil. Steril., 1984, 42, $\mathrm{n}^{\circ} 1$ : 102-107.

\section{ABSTRACT \\ Repercution of chlamydia trachomatis geni- tal infection on semen in male parteners of infertile couples. \\ Ammar-Keskes L, Gdoura R, Bouzid F, Ben Salah.F, Sellami. D, Haktm. H, Hammami A, Rebaï T, Rekik S, Orfila. J}

Chlamydia trachomatis (CT) genital infection is one of the most frequent causes of infertility. Its repercution on semen parameters and male infertility is controversial. The objective of this study was to evaluate the impact of CT genital infection on semen parameters in male partners of infertile couples.

Ninety-seven infertile couples were studied. Semen, urethral and cervical samples were tested for CT by means of direct fluorescence antibodies assay (DFA), cell culture, polymerase chain reaction (PCR) and ELISA. Sera from both parteners were tested for immunoglobulin M, A and $\mathbf{G}$ antibodies to Chlamydia by means of the microimmunofluorescence MIF). For all mens, standard semen parameters were analysed according to the guidlines of the word health organisation.

CT infection was identified in $34 \%$ of the male partners. In $76 \%$ of cases, the infection was asymptomatic. $60,6 \%$ of infected patients's wives were also infected by CT. There was no significant difference between the mean values of concentration, motility and morphology of spermatozoa in both groups of male patients, infected by CT (CT + group) and lacked infection (CT- group). The mean values of motility, vitality, concentration and normal forms of spermatozoa, in both $\mathrm{CT}+$ and CT-groups were respectively: $39,6 \% \pm$ $17,5 \%$ vs $40,4 \% \pm 14,9 \%, 61,9 \% \pm 18,1 \%$ vs
$62,4 \% \pm 18,5 \%, 80,7 \times 10^{6} \pm 67,5 \times 10^{6}$ vs $67,1 \times 106$ $\pm 65,2 \times 10^{6}$ and $34,7 \% \pm 16,7 \%$ vs $33 \% \pm 0,1 \%$. Oligospermia was significantly more frequent in CT+ group $(54,9 \%)$ than in CT- group $(26,9 \%)$. High levels of coiled flagella $(\geq 20)$ were more frequently observed in $\mathrm{CT}+$ group $(18,5 \%)$ than in CT- group $(7,4 \%)$, but the difference was not significant.

We found in this study a high prevalence of genital chlamydial infection into infertile couples. This infection has no repercution on sperm quality, suggesting that there is no effect of CT upon the spermatozoa. But, we can not exclude any impact on fertilisation ability and/or ultrastructure of these gametes. The finding that oligospermia was more frequent in CT+group, leds us to suggest thas chlamydial infection has a repercution on the gametogenesis or on genital ducts permeability. Another hypothesis would be that oligospermia, reflect of spermatogenesis disorder would be associated with reduction of local immunity. Other studies with wide exploration of spermatic functions and of different parts of genital tract are needed to specify the real impact of genital chlamydial infection upon men reproduction function.

Key-Words : Chlamydia trachomatis, genital infection, infertility, sperm analysis. 